\title{
Did we solve soft tissue (breast) attenuation?
}

\author{
Milena J. Henzlova, $M D{ }^{a}$ and W. Lane Duvall, $M D^{b}$ \\ a Department of Cardiology, Mount Sinai Health System, New York, NY \\ b Department of Cardiology, Hartford Hospital, Hartford, CT
}

Received Aug 13, 2019; accepted Aug 14, 2019

doi: 10.1007/s12350-019-01870-1

\section{See related article, pp. 888-897}

Amazingly, in spite of the arrival of newer imaging methods (stress echocardiography, coronary CTA, cardiac MRI), cardiac SPECT myocardial perfusion imaging (MPI) remains the most commonly used noninvasive test for detection of obstructive epicardial coronary artery disease (CAD). The diagnostic and prognostic value of cardiac SPECT has been well studied and documented over the years, intermittently punctuated by the advent of new tracers (Tc-99m sestamibi and Tc-99m tetrofosmin), new camera technology (dual-head and high-efficiency cameras), and new stressors (adenosine and regadenoson).

Regardless of the time period, the limits of SPECT MPI's diagnostic accuracy include both "false negatives" and "false positives." It is now accepted that SPECT perfusion may underestimate the extent of multivessel disease (3 vessel and left main disease). The inclusion of additional clinical data, stress results (e.g., ECG changes, exercise capacity, blood pressure changes, symptoms), gated SPECT data (including left ventricular ejection fraction at rest and post-stress) may decrease the proportion of false negatives and at times alert the clinician to more significant coronary anatomy and physiology than just the "doughnut" pattern. On the opposite end of the spectrum are false positive findings, i.e., the detection of perfusion defects in the absence of anatomical epicardial CAD. Apart from technical reasons (poor equipment QC, patient motion, adjacent tracer activity, and processing errors), the most common source of imaging artifact is the presence of "soft tissue attenuation.', Photons originating in the

Reprint requests: W. Lane Duvall, MD, Hartford Hospital, Hartford, CT; Lane.Duvall@hhchealth.org

J Nucl Cardiol 2021;28:898-900.

$1071-3581 / \$ 34.00$

Copyright (C) 2019 American Society of Nuclear Cardiology. myocardium interact with non-cardiac tissue and are absorbed or scattered prior to their detection by the gamma camera. Crude generalization distinguishes between "diaphragmatic attenuation," more common in male patients (and possibly related to respiratory motion) and "breast attenuation," obviously more common in female patients. The presence of breast implants at times even precludes meaningful evaluation of myocardial perfusion by SPECT imaging because of the significant attenuation artifact created.

Aware of this "Achilles heel" of SPECT imaging, experienced readers use various ways to distinguish between artifactual breast attenuation and real anterior perfusion abnormalities:

1. Size of the bra cup is recorded and is available to the clinical reader, who is alerted to possible interference with overlying soft tissue.

2. Imaging is done with the bra on, both at rest and after stress, to minimize shifts in the breast location.

3. Review of rotating SPECT images, which is a must for all patients, detects patient motion, breast shadow location, and even soft tissue shifts between rest and post-stress imaging sessions. ${ }^{1}$

4. The transition from Tl-201 to Tc-99m agents decreased the prevalence of attenuation artifacts due to higher Tc-99m photon energy and provided the ability to gate the images, allowing for the distinction between scar and attenuation artifact. ${ }^{2}$

5. Attenuation correction using either a transmission source or CT has been introduced and studied, but is not wildly available. ${ }^{3}$

6. If attenuation correction is not available, attenuation reduction using prone or semi-erect imaging is desirable. It improves reader's confidence in separating artifact from a genuine perfusion defect.

7. Interpretation is simplified, if stress images have no anterior defect and rest images do, and wall motion and thickening are preserved: breast attenuation is likely. ${ }^{4}$ Similarly, if the shape of the anterior perfusion defect changes between rest and stress imaging and/or does not follow known coronary 
anatomy, i.e., covers only parts of LAD/D1 territory and breast shadow is visible on rotating images, the experienced reader can interpret with high confidence.

8. Finally, the synthesis of all of the available information, pre-test data, stress data, rest and stress perfusion and gating results, meticulously maintained equipment, high-quality image acquisition and processing, as well as use of the above "tricks of the trade,' allow for best possible clinical interpretation. 5,6

The newest high-efficiency SPECT technology, CZT SPECT, apart from lower tracer dose and shorter imaging time, due to heart centric imaging and markedly increased photon flux, also intuitively promises lesser interference of soft tissue attenuation in female patients. The design of available CZT cameras [two models are available in the USA-Discovery 530 (GE Healthcare) and D-SPECT (Spectrum Dynamics)] includes cardiocentric collimation and stationary detectors; thus all counts are registered simultaneously. With regard to reducing or interpreting attenuation artifact, no rotating images are available with these systems, line source attenuation correction is not available, and the use of CT attenuation correction is not usually practical. The only option for attenuation reduction available is supine and prone imaging (with the Discovery 530) and different degree of semi-recumbent acquisition with the DSPECT camera used in the current study. ${ }^{7}$

In the current issue of the Journal of Nuclear Cardiology, a paper by T. Mannarino et al from Naples, Italy attempts to compare traditional Na-I SPECT (Anger camera) with CZT SPECT (D-SPECT) in female patients. $^{8}$ A total of 109 consecutive women were stressed $(65 \%$ exercised, $45 \%$ underwent dipyridamole vasodilator stress), injected with $370 \mathrm{MBq}(10 \mathrm{mCi})$ of Tc-99m sestamibi and imaged 30 minutes later in random order on both systems (Anger camera-15 minutes of imaging, CZT camera-5 minutes of imaging). Only post-stress gated images were interpreted. Rest imaging may have been done in some patients, but it is unclear in which patients rest imaging was done and what the result was. No attenuation or scatter correction was employed. It is unclear if iterative reconstruction was used for the traditional Na-I SPECT camera data.

All studies were clinically interpreted by two experienced readers who were blinded to the stress test results. A summed stress score $\geq 3$ by semi-quantitative analysis and preserved wall thickening in the anterior wall was considered a "false positive," i.e., consistent with breast attenuation. The clinical read was considered the "'gold standard.' Comparison was made to an automated semi-quantitative result (using Cedar Sinai QGS/QPS program) and also to supervised machine learning analysis. The normalcy rate was calculated: $66 \%(72 / 109)$ of the women were in a low pre-test category. None of the patients included had a high pretest probability or known CAD.

Using the automated semi-quantitative program the authors found a reduction in "false positive" breast attenuation with the CZT camera-24/109 studies were normal using Anger camera, 69/109 were normal using CZT camera; of expected 72 normal studies (patients with low pre-test probability), 58 were abnormal by Anger camera and 29 were abnormal by CZT camera. Overall, by the clinical interpretation, 96/109 (88\%!) tests were normal.

This study suggests a significant advantage of CZT technology over traditional SPECT for the elimination of false positive findings due to breast attenuation. Ultimately, it may be so. However, it is unclear if the readers used all available tools as described above to distinguish between true perfusion abnormalities and artifacts, particularly while imaging using the still globally prevailing traditional Na-I SPECT camera. Perhaps, CZT may be easier technology for an unexperienced reader, and this may be a desirable plus.

Lack of correlation with another modality (either a physiologic or anatomic study) or any prognostic data diminishes the strength of the findings. Clinical reading was used as a "gold standard." However, the readers were blinded to stress results and no-rest imaging was done limiting the validity of the gold standard. The presence of an anterior stress defect and preserved wall thickening were considered consistent with breast attenuation, but the same finding would be present in a case of true anterior ischemia. No-attenuation correction was used, which would have been another opportunity for a "gold standard." Particularly in a study using stress imaging only, some form of attenuation correction or reduction (such as prone imaging or change in recumbent position) is needed. Also, the presented data are derived from a very low risk group of subjects, limiting general applicability.

Perhaps in the future we can look forward to additional CZT camera users more convincingly reproducing and confirming the presented optimistic data.

Until then: Did we solve the soft tissue (breast) attenuation problem? Not yet.

\section{Disclosure}

Milena J. Henzlova and W. Lane Duvall have no conflict of interest related to this manuscript. 


\section{References}

1. Hendel RC, Gibbons RJ, Bateman TM. Use of rotating (cine) planar projection images in the interpretation of a tomographic myocardial perfusion study. J Nucl Cardiol. 1999;6:234-40.

2. Taillefer R, DePuey EG, Udelson JE, Beller GA, Latour Y, Reeves F. Comparative diagnostic accuracy of Tl-201 and Tc-99m sestamibi SPECT imaging (perfusion and ECG-gated SPECT) in detecting coronary artery disease in women. J Am Coll Cardiol. 1997;29:69-77.

3. Berman DS, Kang X, Nishina H, Slomka PJ, Shaw LJ, Hayes SW, et al. Diagnostic accuracy of gated Tc-99m sestamibi stress myocardial perfusion SPECT with combined supine and prone acquisitions to detect coronary artery disease in obese and nonobese patients. J Nucl Cardiol. 2006;13:191-201.

4. DePuey EG, Rozanski A. Using gated technetium-99m-sestamibi SPECT to characterize fixed myocardial defects as infarct or artifact. J Nucl Med. 1995;36:952-5.
5. Cooper JA, Neumann PH, McCandless BK. Effect of patient motion on tomographic myocardial perfusion imaging. $\mathrm{J}$ Nucl Med. 1992;33:1566-71.

6. DePuey EG. Image Artifact. In: Iskandrian AE, Garcia EV, editors. Nuclear cardiac imaging: Principles and applications. 5th ed. New York: Oxford University Press; 2016.

7. Esteves FP, Raggi P, Folks RD, Keidar Z, Askew JW, Rispler S, et al. Novel solid-state-detector dedicated cardiac camera for fast myocardial perfusion imaging: Multicenter comparison with standard dual detector cameras. J Nucl Cardiol. 2009;16:927-34.

8. Mannarino T, Assante R, Ricciardi C, Zampella E, Nappi C, Gaudieri V, et al. Head-to-head comparison of diagnostic accuracy of stress-only myocardial perfusion imaging with conventional and cadmium-zinc telluride single-photon emission computed tomography in women with suspected coronary artery disease. J Nucl Cardiol. 2019. https://doi.org/10.1007/s12350-019-01789-7.

Publisher's Note Springer Nature remains neutral with regard to jurisdictional claims in published maps and institutional affiliations. 